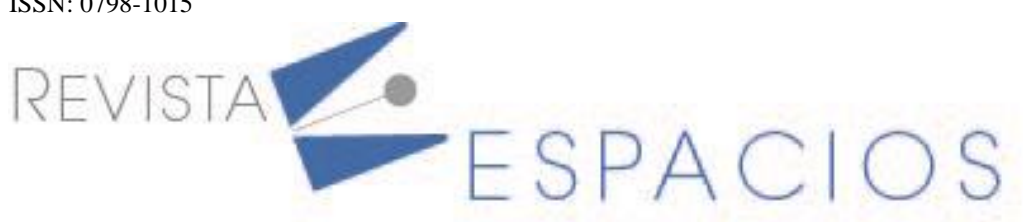

\title{
Herramienta computacional para el análisis y estudio de bifurcaciones en sistemas dinámicos
}

\section{Computational tool for the analysis and study of bifurcations in dynamic systems.}

\begin{abstract}
ROJAS, Beatriz ${ }^{1}$
VALVERDE, José2

Resumen

El presente trabajo consistió en el desarrollo de una herramienta computacional en Matlab, la cual localiza e identifica bifurcaciones en familias uniparamétricas de sistemas dinámicos autónomos discretos y continuos. Constituye un aporte significativo para el estudio de las bifurcaciones uniparamétricas en sistemas dinámicos dado que esta basada en condiciones de bifurcación no estándar, lo cual permite determinar bifurcaciones que otros software existentes no determinan, pues estos están basados en los puntos fijos del sistema, o en condiciones estándar de bifurcación.

Palabras claves: métodos computacionales para bifurcaciones, bifurcaciones, simulación.
\end{abstract}

\begin{abstract}
The present work consisted in the development of a computational tool in Matlab, which locates and identifies bifurcations in uniparametric families of discrete and continuous autonomous dynamic systems. It constitutes a significant contribution to the study of uniparametric bifurcations in dynamic systems since it is based on non-standard conditions of bifurcation which allows to determine bifurcations that other existing software do not determine, since they are based on the system's fixed points or in standard conditions of bifurcation.
\end{abstract}

key words: Computerized methods for bifurcations, bifurcations, simulation.

\section{Introducción}

En el presente trabajo se muestra la herramienta computacional Dsamala Toolbox 2, la cual permite analizar e identificar bifurcaciones locales en familias uniparmétricas de sistemas din micos continuos y discretos. La importancia de esta herramienta comparada con otras existentes es que Dsamala Toolbox-2 se basa en condiciones de bifurcación no estándar y permite determinar bifurcaciones que otras herramientas no logran identificar.

Los sistemas dinámicos han tomado gran importancia en las últimas décadas ya que estos permiten la comprensión y análisis de diversos fenómenos que ocurren en la naturaleza. En muchas ocasiones no es fácil hacer un análisis matemático para identificar y clasificar los cambios presentados en los sistemas cuando éstos sufren pequeñas perturbaciones, dando origen a diferentes tipos de bifurcaciones que se presentan en los sistemas dinámicos autónomos, tanto discretos como continuos. De acuerdo con (Guckenheimer \& Holmes,

\footnotetext{
${ }^{1}$ Grupo de investigación Sistemas Dinámicos, Universidad de los Llanos, Villavicencio-Meta, Colombia. E-mail: brojasg@unillanos.edu.co

2 Universidad Castilla La Mancha, Albacete-España. E-mail: josevalverde@uclm.es
} 
1983) y (Kuznetsov, 2004) la estructura topológica de un sistema puede cambiar al variar el o los parámetros que están presentes en el sistema, es decir, los puntos de equilibrio pueden aparecer, desaparecer o cambiar la estabilidad al variar el valor del o de los parámetros; éstos cambios cualitativos en la dinámica son llamados bifurcaciones. Existen varios tipos de bifurcación entre las cuales podemos mencionar las siguientes:

Bifurcación fold: Sea $f(x, u)$ una familia uniparamétrica, $f(x, u)$ presenta una bifurcación fold en el punto $\left(x_{0}, u_{0}\right)$, si la familia posee una única curva de puntos de equilibrio en el plano determinado por $x, u$, que toca tangencialmente al punto de bifurcación y permanece localmente a un lado de $u_{0}$.

Bifurcación transcritica: Sea $f(x, u)$ una familia uniparamétrica, $f(x, u)$ presenta una bifurcación transcritica en el punto $\left(x_{0}, u_{0}\right)$, si la familia posee dos curvas de puntos de equilibrio en el plano determinado por $x, u$, que pasan a través del origen de dicho plano y que existen localmente a ambos lados del valor paramétrico, cambiando la estabilidad de los puntos fijos al atravesar el valor del parámetro $u_{0}$.

Bifurcación pitchfork: Sea $f(x, u)$ una familia uniparamétrica, $f(x, u)$ presenta una bifurcación pitchfork en el punto $\left(x_{0}, u_{0}\right)$, si la familia posee dos curvas de puntos de equilibrio en el plano determinado por $x, u$; una que pasa a través del origen y que existe localmente a ambos lados de $u_{0}$ y la otra que toca tangencialmente al origen, permaneciendo a uno de los lados de $u_{0}$.

Bifurcación flip: Sea $f(x, u)$ una familia uniparamétrica, $f(x, u)$ presenta una bifurcación flip en el punto $\left(x_{0}, u_{0}\right)$, si la familia posee dos curvas en el plano determinado por las variables $x, u$; una curva de puntos de periodo dos que toca tangencialmente al punto de bifurcación permaneciendo a uno de los lados de $u_{0}$ y la otra de puntos fijos que lo atraviesan.

A continuación se enuncian las condiciones estándar de bifurcación en familias uniparamétricas.

Sea $f(x, u)$ una familia uniparamétrica de sistemas discretos de clase $C^{2}$, que presenta en $u_{0}=0$ el punto fijo $x_{0}=0$.

Si $f_{x}\left(x_{0}, u_{0}\right)=1, f_{x x}\left(x_{0}, u_{0}\right) \neq 0, f_{u}\left(x_{0}, u_{0}\right) \neq 0$, entonces $f(x, u)$ presenta una bifurcación fold.

Si $f_{x}\left(x_{0}, u_{0}\right)=1, f_{u}\left(x_{0}, u_{0}\right)=0, f_{x x}\left(x_{0}, u_{0}\right) \neq 0$ y $f_{x u}\left(x_{0}, u_{0}\right) \neq 0$, entonces $f(x, u)$ presenta una bifurcación transcritica.

Si $f_{x}\left(x_{0}, u_{0}\right)=1, f_{u}\left(x_{0}, u_{0}\right)=0, f_{x x x}\left(x_{0}, u_{0}\right) \neq 0$ y $f_{x u}\left(x_{0}, u_{0}\right) \neq 0$, entonces $f(x, u)$ presenta una bifurcación pichfork.

Si $f_{x}\left(x_{0}, u_{0}\right)=-1, f_{u}\left(x_{0}, u_{0}\right)=0, f^{2}{ }_{x x x}\left(x_{0}, u_{0}\right) \neq 0$ y $f_{x u}\left(x_{0}, u_{0}\right) \neq 0$, entonces $f(x, u)$ presenta una bifurcación flip.

Este artículo se ha estructurado de la siguiente manera: En la sección 2 se describen los antecedentes sobre las herramientas computacionales existentes para el estudio de sistemas dinámicos, en especial las bifurcaciones. Se enuncian las condiciones de bifurcación no estándar para las bifurcaciones: fold, transcritica, pitchfork y flip, tanto, para sistemas continuos como para sistemas discretos. En la sección 3, se presentan los preliminares y la metodología a seguir para la creación de la herramienta computacional. En la siguiente sección, se presentan los resultados obtenidos, dando ejemplos de algunas bifurcaciones que presentan en sistemas discretos y en sistemas continuos. Finalmente, en la sección 5 se presentan las principales conclusiones del trabajo. 


\section{Antecedentes}

Existen algunas herramientas computacionales para el estudio de bifurcaciones en sistemas dinámicos autónomos discretos o continuos; las cuales se basan en el análisis de la estabilidad de los puntos fijos del sistema o en condiciones estándar de bifurcación. Algunas de ellas presentan un entorno gráfico complejo lo que dificulta su uso o están diseñadas sólo para una aplicación particular. A continuación se describen algunos software existentes para el estudio de sistemas dinámicos.

- El IGB: Identificador grafico (Tovar, 2000). Desarrollado en la plataforma de MATLAB, el cual localiza e identifica bifurcaciones locales en sistemas din micos continuos y discretos, aut nomos, multiparam tricos y multidimensionales; dentro de las principales caracter sticas de este se encuentra su capacidad de identificar directamente las bifurcaciones fold y Hopf para sistemas din micos continuos, y fold, flip y Neimark-Sacker para sistemas din micos discretos, su interfaz gr fica es complicado para un usuario sin experiencia en MATLAB.

- Java PHASER (Glick and Kocak) simula sistemas din micos discretos y continuos utilizando variables reales. No es software libre.

- MATDS (Govorukhin). Es software basado en MATLAB para la investigaci $n$ de los sistemas din micos el cual muestra las bifurcaciones y los exponentes de Lyapunov, usa $\mathrm{m}$ todos de an lisis de sistemas y su interfaz gr fica es un poco compleja.

- Pplane y dfield (Polking and Arnold) generan y muestran retratos de fase de un sistema din mico. Tiene funciones $b$ sicas para la simulaci $n$ de un sistema, pero no puede analizar completamente un sistema din mico que se encuentra en las versiones de Java y MATLAB.

- MATCONT(Govaerts and Kuznetsov, 2010) paquete de gr ficos de MATLAB para el estudio num rico interactivo de los sistemas din micos, que se especializa en el an lisis de las bifurcaciones.

- DDE - BITFTOOL (Luzyanina, Samaey, roose and Verheyden, 2010) paquete de MATLAB para la bifurcaci $n$ num rica y el an lisis de la estabilidad de las ecuaciones diferenciales de retardo.

- System-Solver (Dom nguez, Ardilla and Bustamante, 2010) herramienta de c digo abierto para el modelado de sistemas din micos en Java y se centra en la soluci $n$ num rica de sistemas din micos deterministas utilizando el $m$ todo de Runge- Kutta; $s$ lo tiene esa funci $n$, su ventaja radica en la soluci $n$ de sistemas de ecuaciones de cualquier orden y exportar datos a otros lenguajes de programaci $n$.

- Dsamala (Atehort a, Ladino y Valverde, 2012). Herramienta que tiene una variedad de caracter sticas, como su interfaz gr fica de usuario, es simple y $f$ cil de usar y tiene un conjunto de funciones (caja de herramientas ) en el que el usuario puede controlar el num rica c digo desarrollado, lo que permite un mejor an lisis y simulaci $\mathrm{n}$ de un sistema din mico. La desventaja es que no analiza bifurcaciones de sistemas din micos. Dsamala presenta una flexible arquitectura modular, permitiendo la incorporación de nuevas funcionalidades, en nuestro caso las bifurcaciones.

\subsection{Condiciones de bifurcación no estándar}

En Balibrea, Martinez \& Valverde (2010) se determinan condiciones no estándar que permiten encontrar bifurcaciones en familas uniparamétricas de sistemas autónomos discretos y continuos, (ver también en Balibrea \& Valverde, 2001; Balibrea \& Valverde, 2003). En Elaydi, Luís, y Oliveira (2013) se determinan condiciones de segundo orden, las cuales garantizan la presencia de bifurcación de tipo fold, trascritica y pitchfork en familias multiparamétricas. Balibrea, Oliveira y Valverde (2017) demostran la equivalencia topológica entre familias de funciones uniparamétricas que experimentan el mismo tipo de bifurcación.

Para realizar los algoritmos que permitan la incorporación de una nueva funcionalidad a Dsamala que permita el análisis de bifurcaciones en familias uniparamétricas es necesario conocer, estudiar y analizar los teoremas que generalizan las condiciones de bifurcación no estándar, tanto en sistemas discretos como en sistemas continuos (condiciones de orden superior).

Teorema: Condición no estándar de bifurcación 
Sea $f(x, u)$ una familia uniparamétrica de sistemas discretos de clase $C^{2 n}$, que presenta en la función correspondiente al valor paramétrico $u_{0}$ el punto fijo $x_{0}$.

1) $f(x, u)$ presenta una bifurcación fold, si $f(x, u)$ satisface las siguientes condiciones:

i. $f_{x}\left(x_{0}, u_{0}\right)=1$,

ii. $\quad f_{x x}\left(x_{0}, u_{0}\right)=f_{x x x}\left(x_{0}, u_{0}\right)=\cdots=f_{x^{2 n-2}}\left(x_{0}, u_{0}\right)=0$,

iii. $f_{x^{2 n}}\left(x_{0}, u_{0}\right) \neq 0$,

iv. $f_{u}\left(x_{0}, u_{0}\right) \neq 0$.

2) $f(x, u)$ presenta una bifurcación transcrita, cuando $f(x, u)$ satisface :

i. $f_{x}\left(x_{0}, u_{0}\right)=1$,

ii. $f_{u}\left(x_{0}, u_{0}\right)=0$,

iii. $\quad f_{x x}\left(x_{0}, u_{0}\right)=f_{x x x}\left(x_{0}, u_{0}\right)=\cdots=f_{x^{2 n-1}}\left(x_{0}, u_{0}\right)=0$,

iv. $f_{x^{2 n}}\left(x_{0}, u_{0}\right) \neq 0$,

v. $f_{x u}\left(x_{0}, u_{0}\right) \neq 0$.

3) $f(x, u)$ presenta una bifurcación tipo pichfork, si se cumplen las siguientes condiciones:

i. $f_{x}\left(x_{0}, u_{0}\right)=1$,

ii. $f_{u}\left(x_{0}, u_{0}\right)=0$,

iii. $f_{x x}\left(x_{0}, u_{0}\right)=f_{x x x}\left(x_{0}, u_{0}\right)=\cdots=f_{x^{2 n}}\left(x_{0}, u_{0}\right)=0$,

iv. $f_{x^{2 n+1}}\left(x_{0}, u_{0}\right) \neq 0$,

v. $f_{x u}\left(x_{0}, u_{0}\right) \neq 0$.

4) Finalmente, $f(x, u)$ presenta una bifurcación flip, si $f(x, u)$ satisface:

i. $f_{x}\left(x_{0}, u_{0}\right)=-1$,

ii. $f_{x x x}^{2}\left(x_{0}, u_{0}\right)=f^{2}{ }_{x^{4}}\left(x_{0}, u_{0}\right)=\cdots=f^{2}{ }_{x^{2 n}}\left(x_{0}, u_{0}\right)=0$,

iii. $f^{2} x^{2 n+1}\left(x_{0}, u_{0}\right) \neq 0$,

iv. $f_{x u}\left(x_{0}, u_{0}\right) \neq 0$.

En los sistemas autónomos continuos se extienden las mismas condiciones. Sea $f(x, u)$ una familia uniparamétrica de sistemas continuos de clase $C^{2 n}$, que presenta en la función corrrespondiente al valor paramétrico $u_{0}$ el punto de equilibrio $x_{0}$.

1) $f(x, u)$ presenta una bifurcación fold, si $f(x, u)$ satisface las siguientes condiciones:

i. $f_{x}\left(x_{0}, u_{0}\right)=0$,

ii. $\quad f_{x x}\left(x_{0}, u_{0}\right)=f_{x x x}\left(x_{0}, u_{0}\right)=\cdots=f_{x^{2 n-2}}\left(x_{0}, u_{0}\right)=0$,

iii. $f_{x^{2 n}}\left(x_{0}, u_{0}\right) \neq 0$,

iv. $f_{u}\left(x_{0}, u_{0}\right) \neq 0$.

2) $f(x, u)$ presenta una bifurcación transcrita, cuando $f(x, u)$ satisface :

i. $f_{x}\left(x_{0}, u_{0}\right)=0$,

ii. $f_{u}\left(x_{0}, u_{0}\right)=0$,

iii. $f_{x x}\left(x_{0}, u_{0}\right)=f_{x x x}\left(x_{0}, u_{0}\right)=\cdots=f_{x^{2 n-1}}\left(x_{0}, u_{0}\right)=0$,

iv. $f_{x^{2 n}}\left(x_{0}, u_{0}\right) \neq 0$,

v. $f_{x u}\left(x_{0}, u_{0}\right) \neq 0$.

3) $f(x, u)$ presenta una bifurcación tipo pichfork, si se cumplen las siguientes condiciones:

i. $f_{x}\left(x_{0}, u_{0}\right)=1$,

ii. $f_{u}\left(x_{0}, u_{0}\right)=0$,

iii. $f_{x x}\left(x_{0}, u_{0}\right)=f_{x x x}\left(x_{0}, u_{0}\right)=\cdots=f_{x^{2 n}}\left(x_{0}, u_{0}\right)=0$, 
iv. $f_{x^{2 n+1}}\left(x_{0}, u_{0}\right) \neq 0$,

v. $f_{x u}\left(x_{0}, u_{0}\right) \neq 0$.

\section{Metodología}

Para el desarrollo de Dsamala Toolbox-2 se utilizó la metodología de desarrollo basada en funcionalidades (FDD Feature Driven Development). En la primera etapa se estudiaron las condiciones de bifurcaciones no estándar en familias uniparamétricas encontradas por Balibrea et al. (2010). Luego se analizó la herramienta Dsamala Toolbox (Atehortúa et al. 2012), la cual consiste en un conjunto de funciones en Matlab que permite el análisis y simulación de sistemas dinámicos discretos y continuos, tiene la ventaja que cualquier usuario puede interactuar fácilmente con ella a través de una interfaz de usuario muy amigable.

En una segunda etapa se determinó las bifurcaciones a incluir en la herramienta computacional. Para el caso discreto, se trabajó con las bifurcación: fold, transcritica, pitchfork y para el caso continuo se utilizó la bifurcción: fold, transcritica, pitchfork. Posterior a ello se diseñarón los algoritmos numéricos para identificar las bifurcaciones seleccionadas; estos algoritmos se basarón en condiciones de bifurcación no estándar.

Seguidamente, teniendo en cuenta la flexible arquitectura modular de Dsamala Toolbox, se incorporó una nueva funcionalidad a esta, para nuestro caso el análisis de bifurcaciones. Así se dio creación a Dsamala Toolbox-2, la cual permite determinar bifurcaciones que otros software en el mercado no determinan; puesto que se basan en condiciones de bifurcación estándar. Finalmente se válido la herramienta, para ello se comparó los resultados del análisis de bifurcación por medio de Dsamala Toolbox-2 con los resultados obtenidos analíticamente.

\section{Resultados}

Se ha creado la herramiena Dsamala Toolbox 2, la cual permite determinar en sistemas discretos, si una familia uniparamétrica presenta bifurcación de tipo: fold, transcritica, pitchfork o flip y en sistemas continuos si una familia uniparamétrica presenta bifurcación de tipo: fold, transcritica o pitchfork.

\subsection{Análisis bifurcaciones en sistemas discretos.}

Para hallar los puntos de equilibrio de cada una de las familias uniparamétricas $f(x, u)$ presentadas a lo largo del trabajo se evalúa los puntos fijos en términos de $x$ a través de la asignación de valores al parámetro $u$ dentro del rango [-2,2] con incrementos de 0,01. Este rango de incremento es utilizado en todos los ejemplos de este artículo.

La familia uniparamétrica $f(x, u)=x^{2}+u$, presenta una bifurcación fold en el punto de equilibrio $x=0,5$ con $u=0,25$. En la Figura 1 se visualiza la interfaz gráfica obtenida utilizando el software Dsamala Toolbox-2. 
Figura 1

Gráfica de la estabilidad

del sistema $f(x, u)=x^{2}+u$

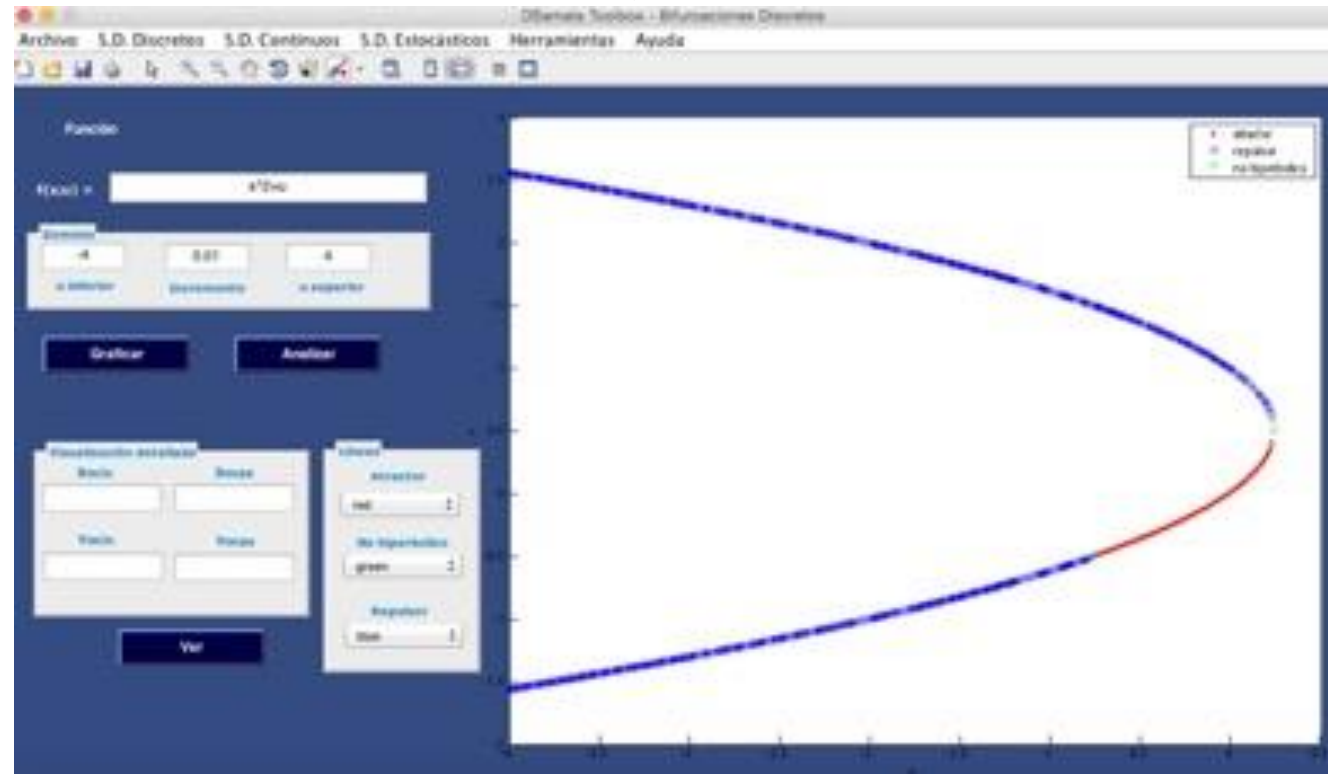

Fuente: Dsamala Toolbox-2

La figura 2 muestra el resultado del análisis de bifurcación del sistema $f(x, u)=x^{2}+u$ usando Dsamala Toolbox-2, se evidencia que dicha función presenta una bifurcación fold en el punto de equilibrio $x=0,5$ en el valor parámetro $=0,25$. El diagrama de bifurcación de este sistema se muestra en la Figura 3.

Figura 2

Descripción del análisis de bifurcación

obtenido del sistema $f(x, u)=x^{2}+u$

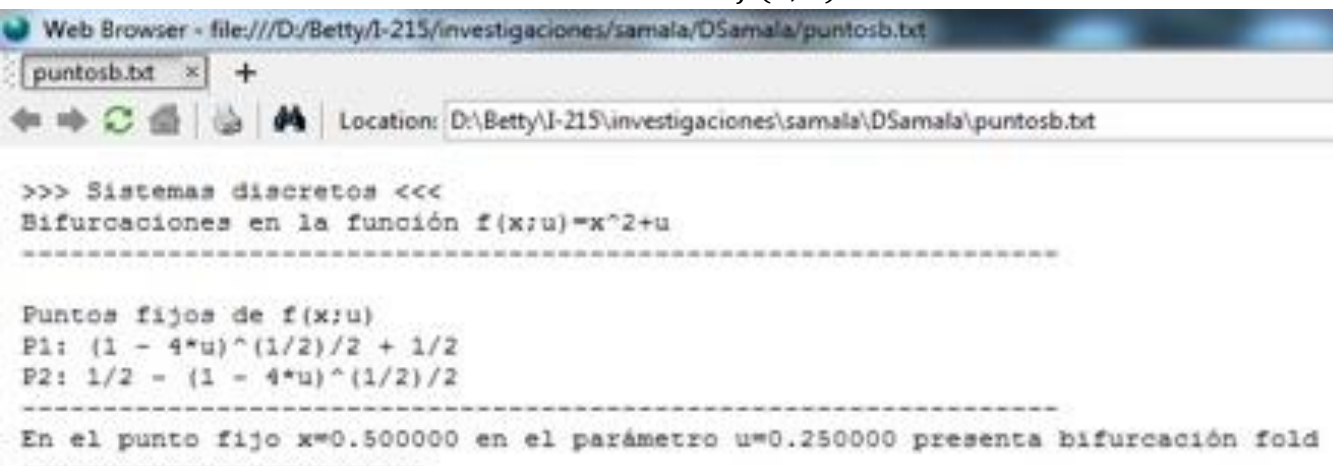

Fuente: Dsamala Toolbox-2 
Figura 3

Diagrama de bifurcación obtenido del sistema $f(x, u)=x^{2}+u$

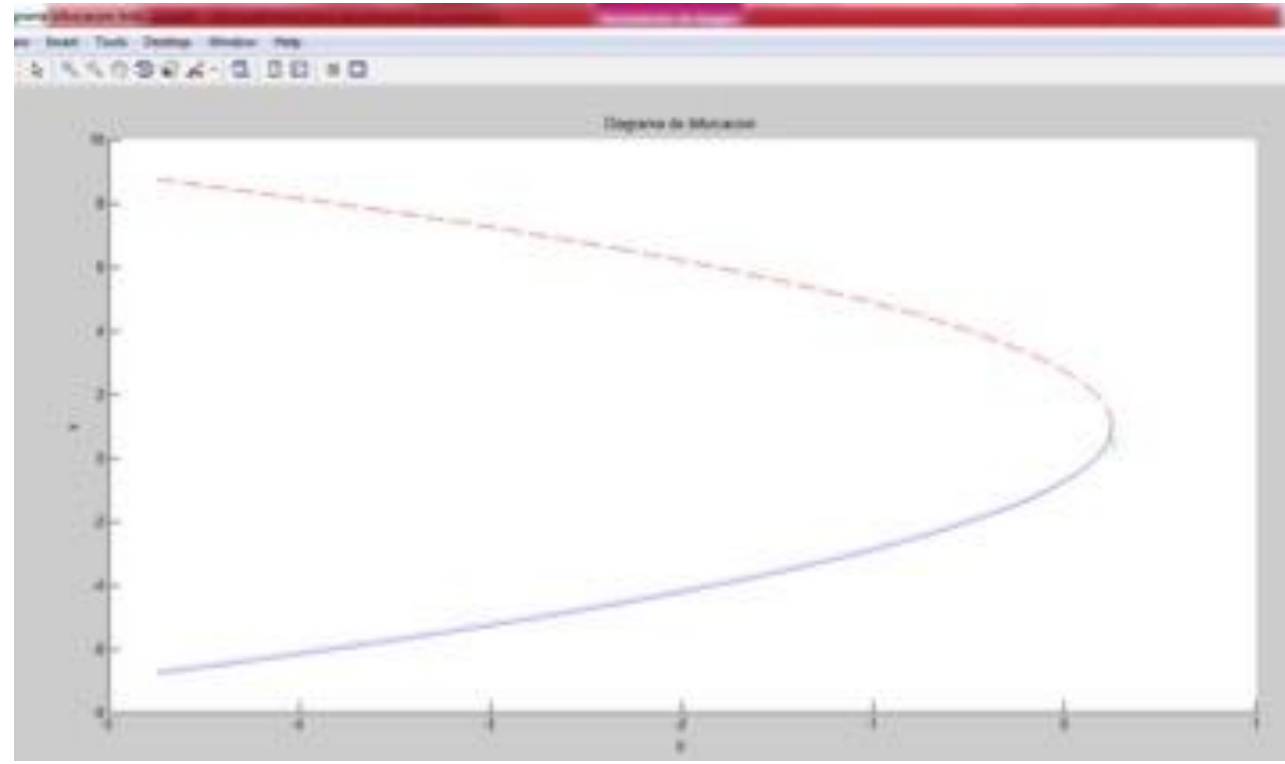

Fuente: Dsamala Toolbox-2

La función $f(x, u)=-x-u x+x^{3}$ presenta una bifurcación pitchfork en el punto de equilibrio $x=0$, con parámetro $u=-2$ y una bifurcación flip en el punto de equilibrio $x=0$ con parámetro $u=0$. En la Figura 4 se muestra la interfaz gráfica para analizar este sistema en el software Dsamala-2, y en la Figura 5 se visualiza el análisis del tipo de bifurcación. La Figura 6 y Figura 7 muestran el diagrama de bifurcación del sistema.

Figura 4

Interfaz gráfica de $f(x, u)=-x-u x+x^{3}$

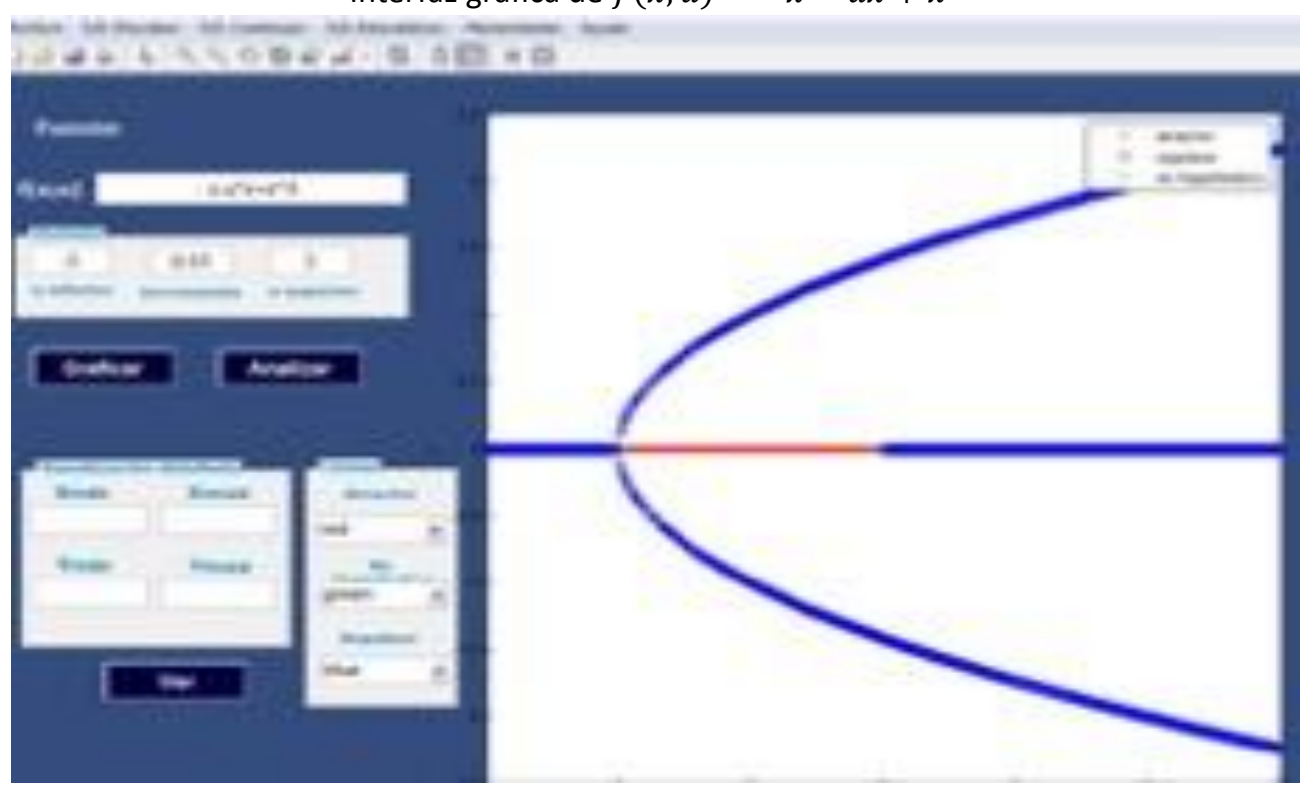

Fuente: Dsamala Toolbox-2 
Figura 5

Descripción del análisis de bifurcación

del sistema $f(x, u)=-x-u x+x^{3}$

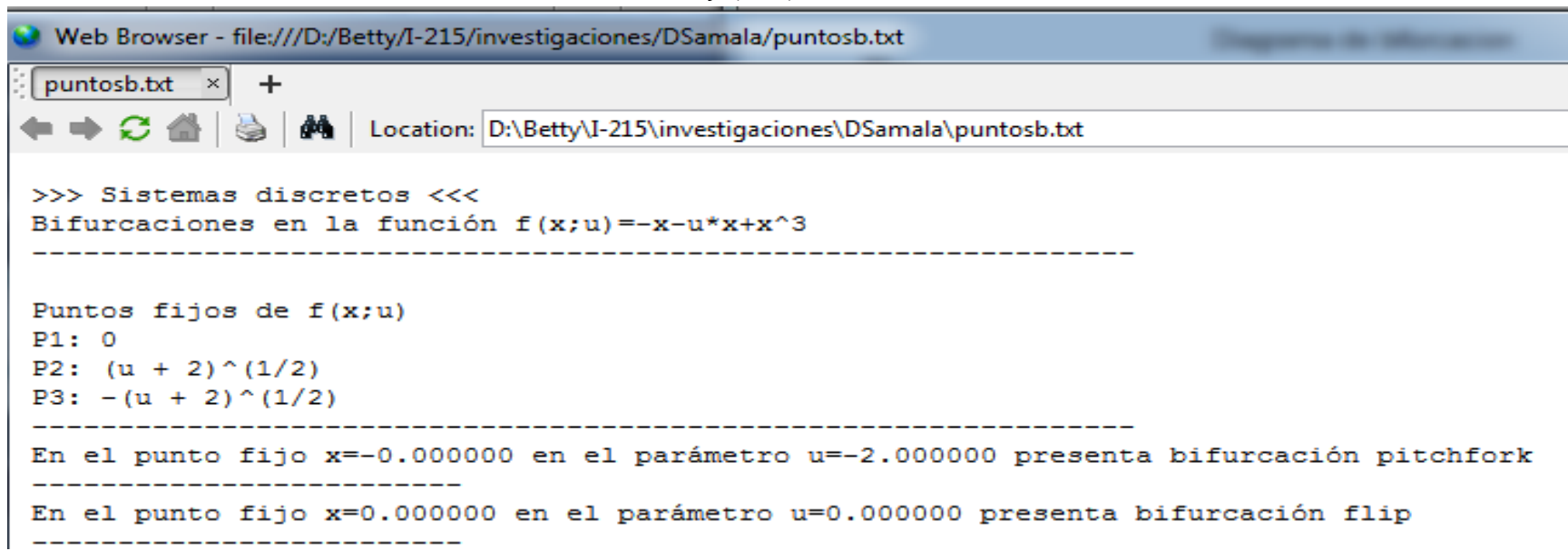

Fuente: Dsamala Toolbox-2

Figura 6

Diagrama de bifurcación pitchfork para $f(x, u)=-x-u x+x^{3}$

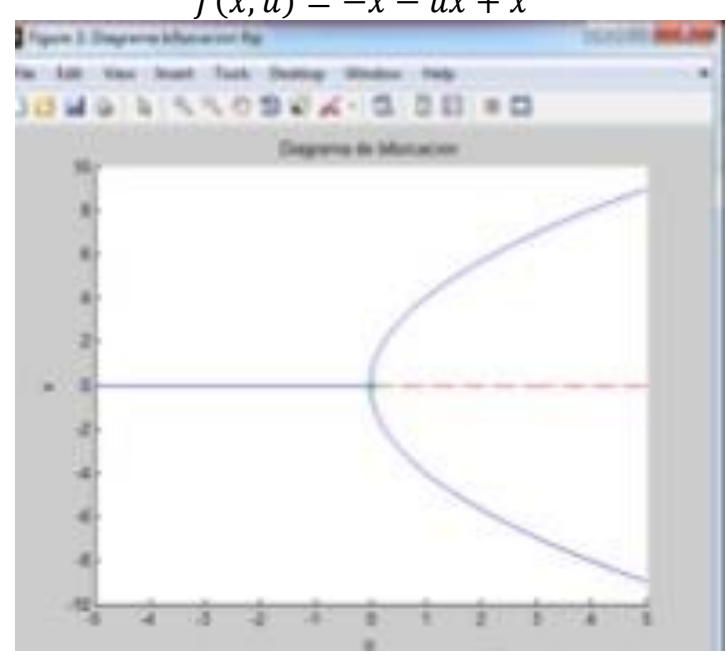

Fuente: Dsamala Toolbox-2
Figura 7

Diagrama de bifurcación flip para

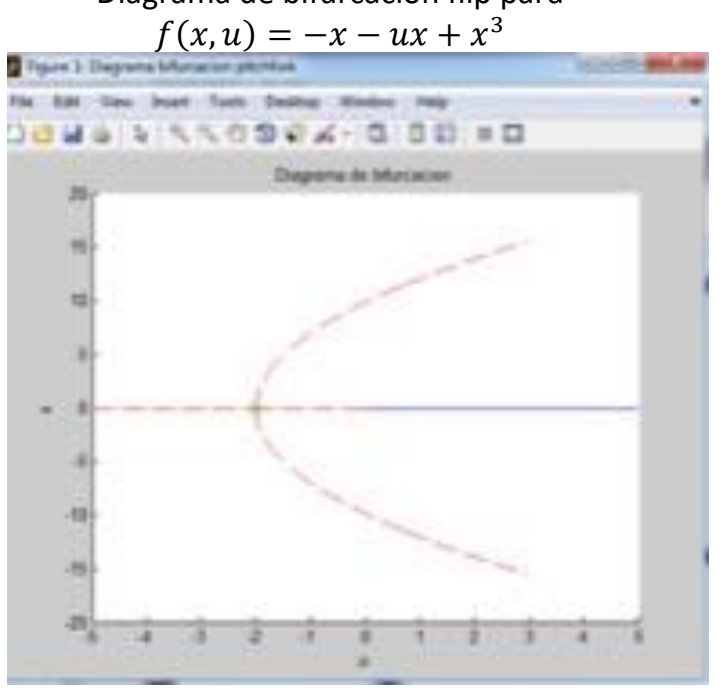

Fuente: Dsamala Toolbox-2

El sistema $f(x, u)=x+u x+x^{2}$ presenta una bifurcación transcritica en el punto de equilibrio 0 , bajo el paraméto $u=0$. La Figura 8 muestra la interfaz gráfica para la función dada, la Figura 9 presenta el resultado del análisis de la bifurcación que sufre el sistema y la Figura 10 presenta el diagrama de bifurcación. 
Figura 8

Interfaz gráfica de la función $f(x, u)=x+u x+x^{2}$

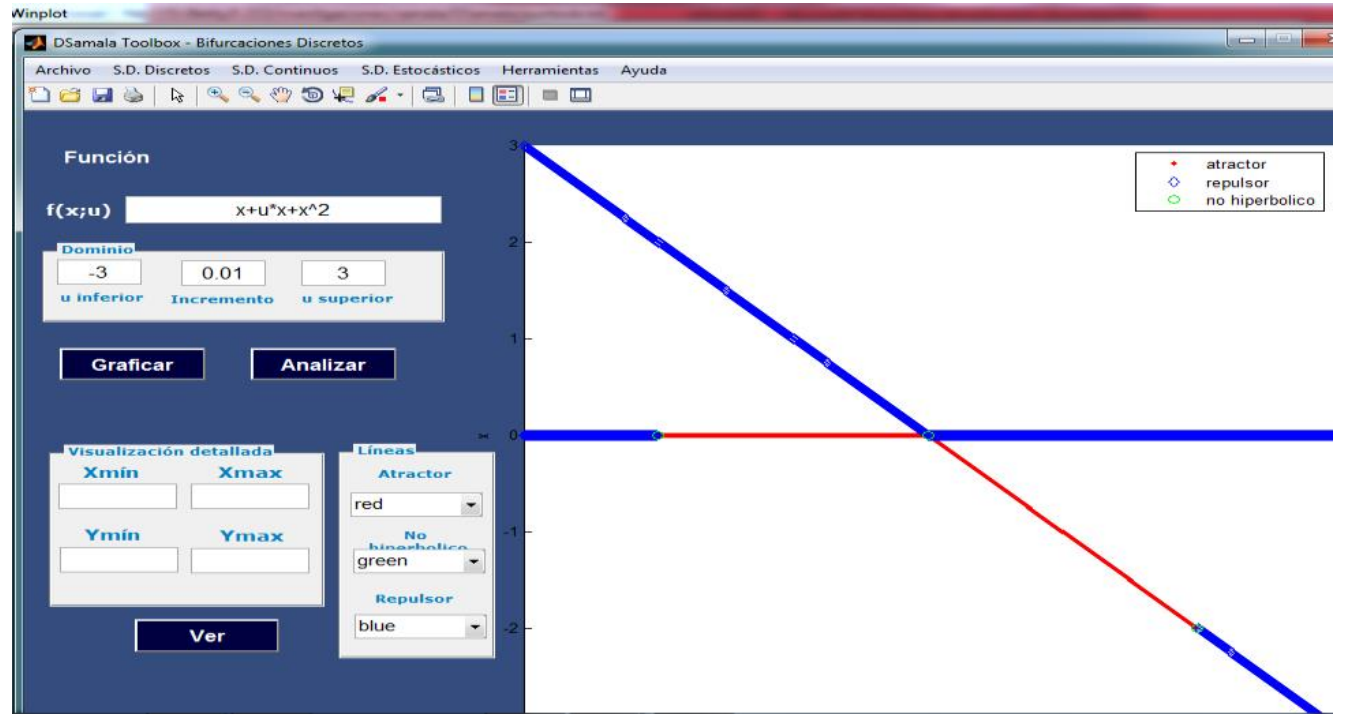

Fuente: Dsamala Toolbox-2

Figura 9

Descripción del análisis de bifurcación obtenida del sistema $f(x, u)=x+u x+x^{2}$

Web Browser - file:///D:/Betty/1-215/investigaciones/samala/DSamala/puntosb.txt

puntosb.txt $x+$

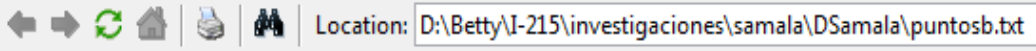

$\gg>$ Sistemas discretos $<<<$

Bifurcaciones en la función $\mathrm{f}(\mathrm{x} ; \mathrm{u})=\mathrm{x}+\mathrm{u}^{\star} \mathrm{x}+\mathrm{x}^{\wedge} 2$

Puntos fijos de $f(x ; u)$

P1: 0

P2: $-\mathrm{u}$

$----$

En el punto fijo $\mathrm{x}=-0.000000$ en el parámetro u=0.000000 presenta bifurcación transcrítica

Fuente: Dsamala Toolbox-2

Figura 10

Diagrama de bifurcación transcritica de la función $f(x, u)=x+u x+x^{2}$

Figure 1: Diagrama bifurcacion transcritica

File Edit View Insert Tools Desktop Window Help

Diagrama de bifurcacion

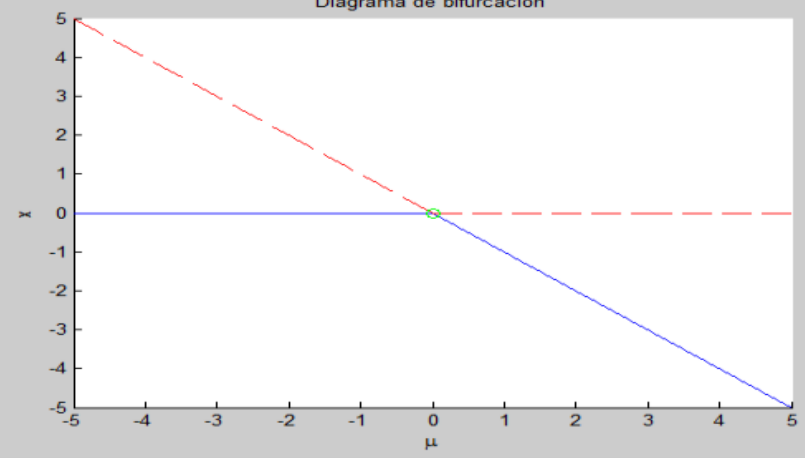

Fuente: Dsamala Toolbox-2 
El sistema $f(x, u)=x+u-x^{2}$ presenta una bifurcación fold en el punto de equilibrio 0 , bajo el paraméto $u=0$. En la Figura 11 se presenta el resultado del análisis de la bifurcación y en la Figura 12 se muestra el diagrama de bifurcación para la familia uniparamétrica $f(x, u)=x+u-x^{2}$.

Figura 11

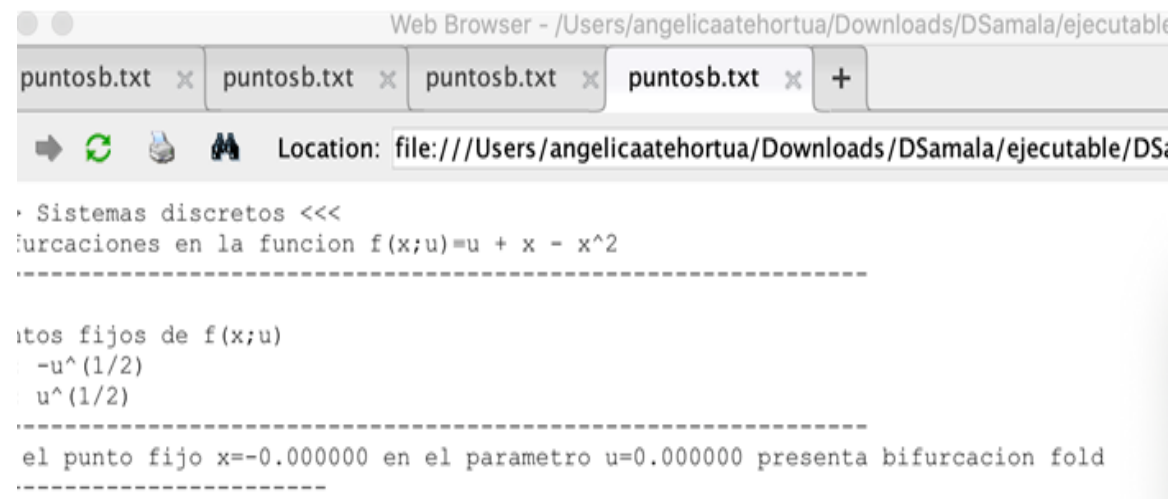

Fuente: Dsamala Toolbox-2

\section{Figura 12}

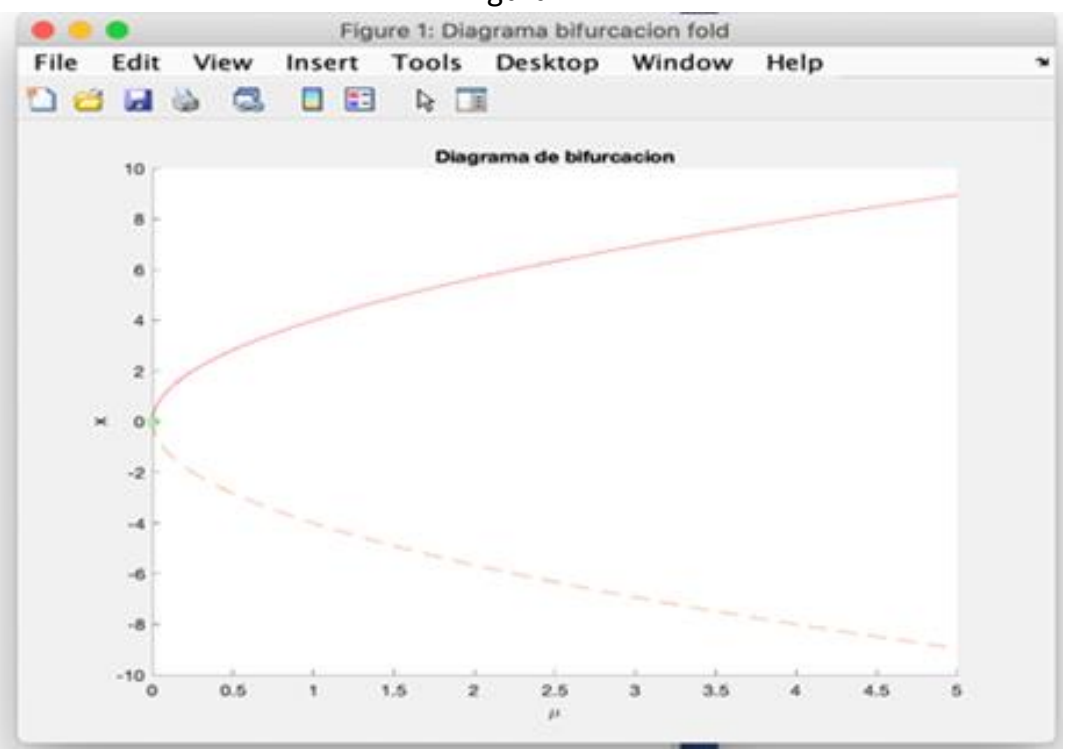

Fuente: Dsamala Toolbox-2

El sistema uniparamtrico $f(x, u)=u x-x^{3}$ presenta una bifurcación pitchfork en el punto de equilibrio 0 , bajo el paraméto $u=1$ y una bifurcación flip en el punto fijo $x=0$, bajo el paramétro $u=-1$. En la Figura 13 se presenta el resultado del análisis de la bifurcación . 
Figura 13

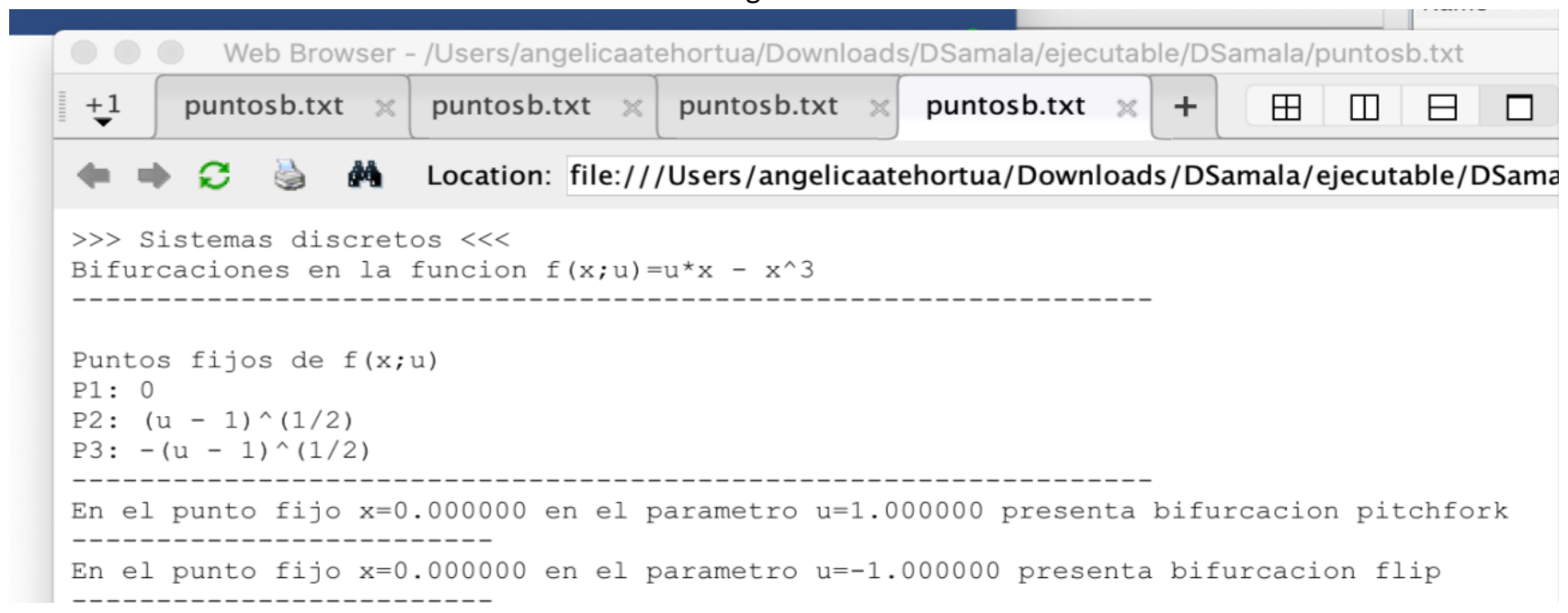

Fuente: Dsamala Toolbox-2

\subsection{Análisis bifurcaciones en sistemas continuos.}

A continuaci $n$ se presentan algunos ejemplos de bifurcaciones presentadas en sistemas continuos.

El sistema uniparamétrico $f(x, u)=u-x^{2}$ presenta una bifurcaci $\mathrm{n}$ fold en el par metro $u=-\frac{1}{2}$ con el punto de equilibrio $x_{0}=\frac{1}{2}$. En la Figura 14 se presenta la interfaz gráfica del sistema; en la Figura 15 se evidencia la bifurcación fold que sufre el sistema al atravesar el valor paramétrico $u=-\frac{1}{2}$ con el punto de equilibrio $x_{0}=\frac{1}{2}$. La Figura 16 muestra el diagrama de bifurcación.

Figura 14

Interfaz gráfica de la función $f(x, u)=u-x^{2}$

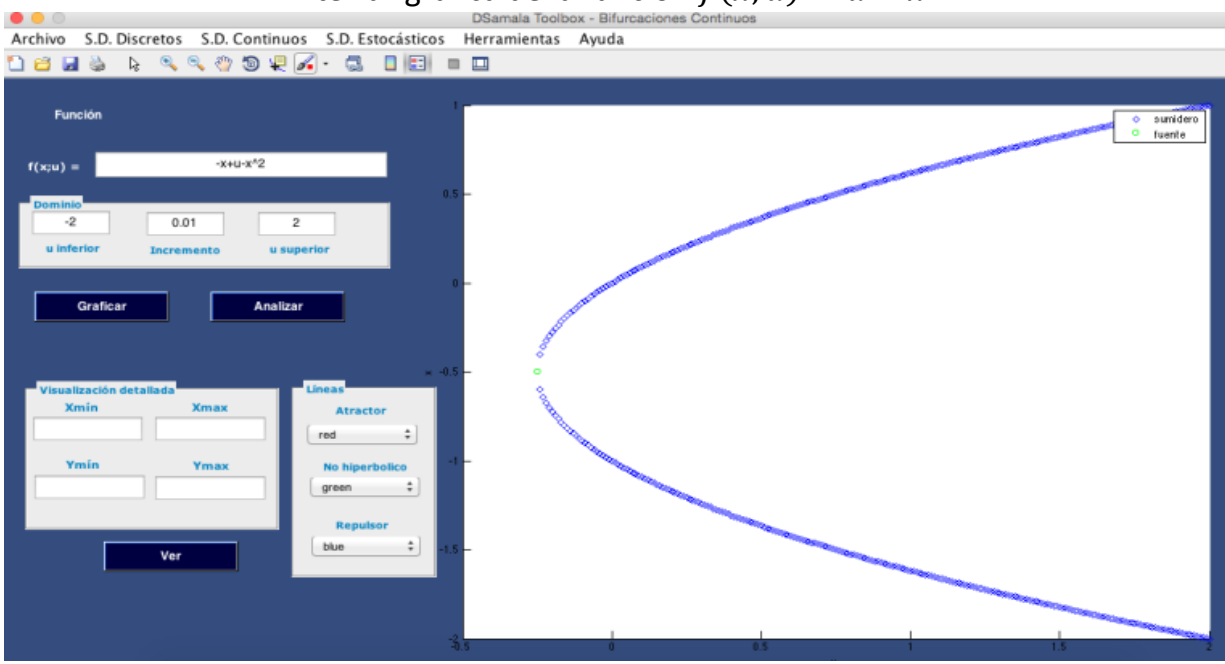

Fuente: Dsamala Toolbox-2 
Figura 15

Descripción del análisis de bifurcación obtenida del sistema $f(x, u)=u-x^{2}$

Web Browser - file:///D:/Betty/1-215/investigaciones/samala/DSamala/puntosb.txt

puntosb.txt $x+$

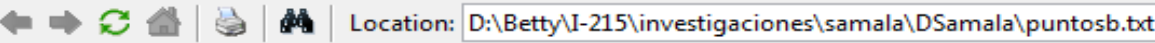

$\gg$ Sistemas continuos unidimensionales $\ll<$

Bifurcaciones en la función $f(x ; u)=-x+u-x \wedge 2$

Puntos fijos de $f(x ; u)$

P1: $(4 * u+1) \wedge(1 / 2) / 2-1 / 2$

P2: $-(4 * u+1) \wedge(1 / 2) / 2-1 / 2$

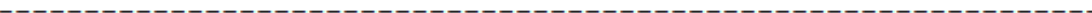

En el punto fijo $\mathrm{x}=-0.500000$ en el parámetro u=-0.250000 presenta bifurcación fold

Fuente: Dsamala Toolbox-2

Figura 16

Diagrama de bifurcación de la función $f(x, u)=u-x^{2}$

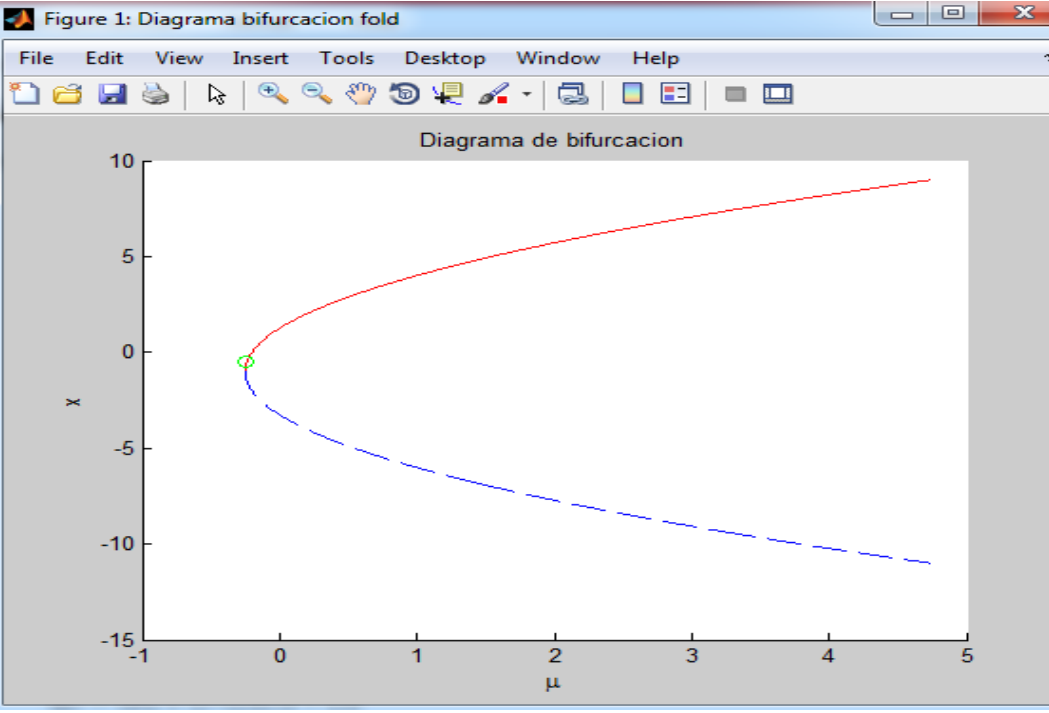

Fuente: Dsamala Toolbox-2

El sistema $f(x, u)=x^{2}+u x$ presenta una bifurcaci $\mathrm{n}$ transcritica en el punto de equilibrio $x=0$ bajo el par metro $u=0$. La Figura 17 muestra la interfaz gráfica de la función usando Dsamala Toolbox-2. EL análisis de bifurcación del sistema utilizando Dsamala Toolbox-2 se puede observar en las Figura 18. La Figura 19 muestra el diagrama de bifurcación de $f$. 
Figura 17

Interfaz gráfica de la función $f(x, u)=x^{2}+u x$

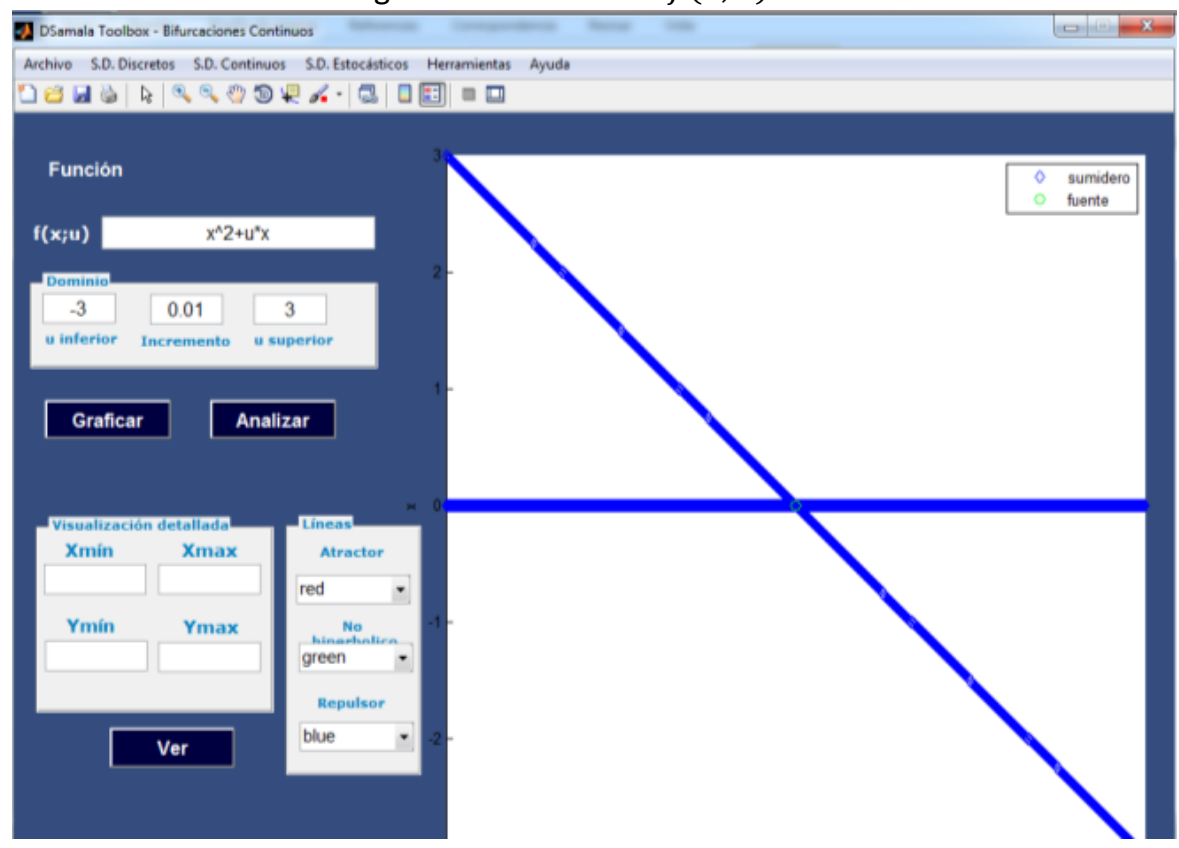

Fuente: Dsamala Toolbox-2

Figura 18

Descripción del análisis de bifurcación del sistema $f(x, u)=x^{2}+u x$

Web Browser - file:///puntosb.txt

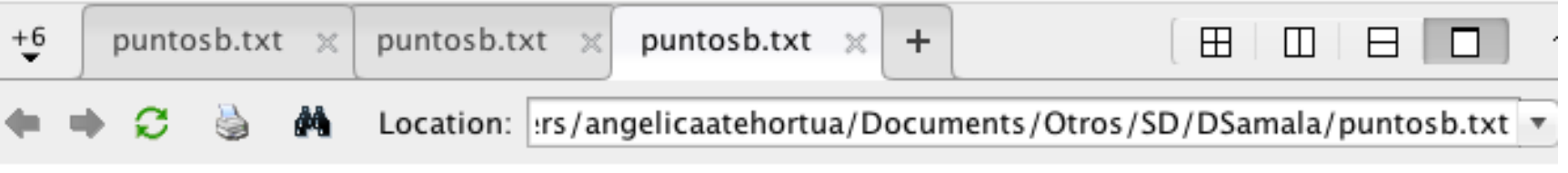

\> Sistemas continuos unidimensionales $\ll$

Bifurcaciones en la función $f(x ; u)=x^{\wedge} 2+u * x$

Puntos fijos de $f(x ; u)$

P1: 0

P2: $-\mathrm{u}$

En el punto fijo $\mathbf{x}=0.000000$ en el parámetro $u=0.000000$ presenta bifurcación transcrítica

Fuente: Dsamala Toolbox-2 
Figura 19

Diagrama de bifurcación de la función $f(x, u)=x^{2}+u x$

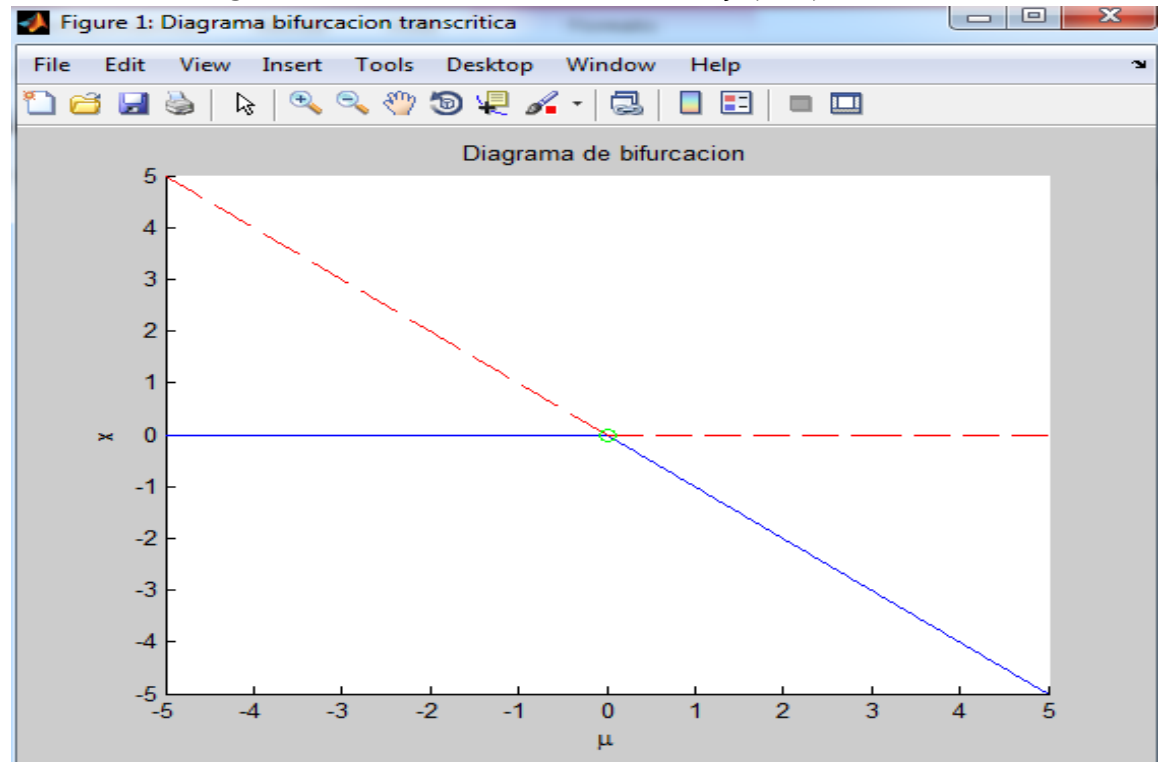

Fuente: Dsamala Toolbox-2

La Figura 20 muestra la interfaz gráfica para el sistema $f(x, u)=x^{3}+u x$, En la figura 21 se evidencia que presenta una bifurcaci $\mathrm{n}$ pitchfork en el punto de equilibrio $x=0$, bajo el par metro $u=-2$. En la Figura 22 se muestra el diagrama de bifurcación.

Figura 20

Interfaz gráfica de la función $f(x, u)=x^{3}+u x$

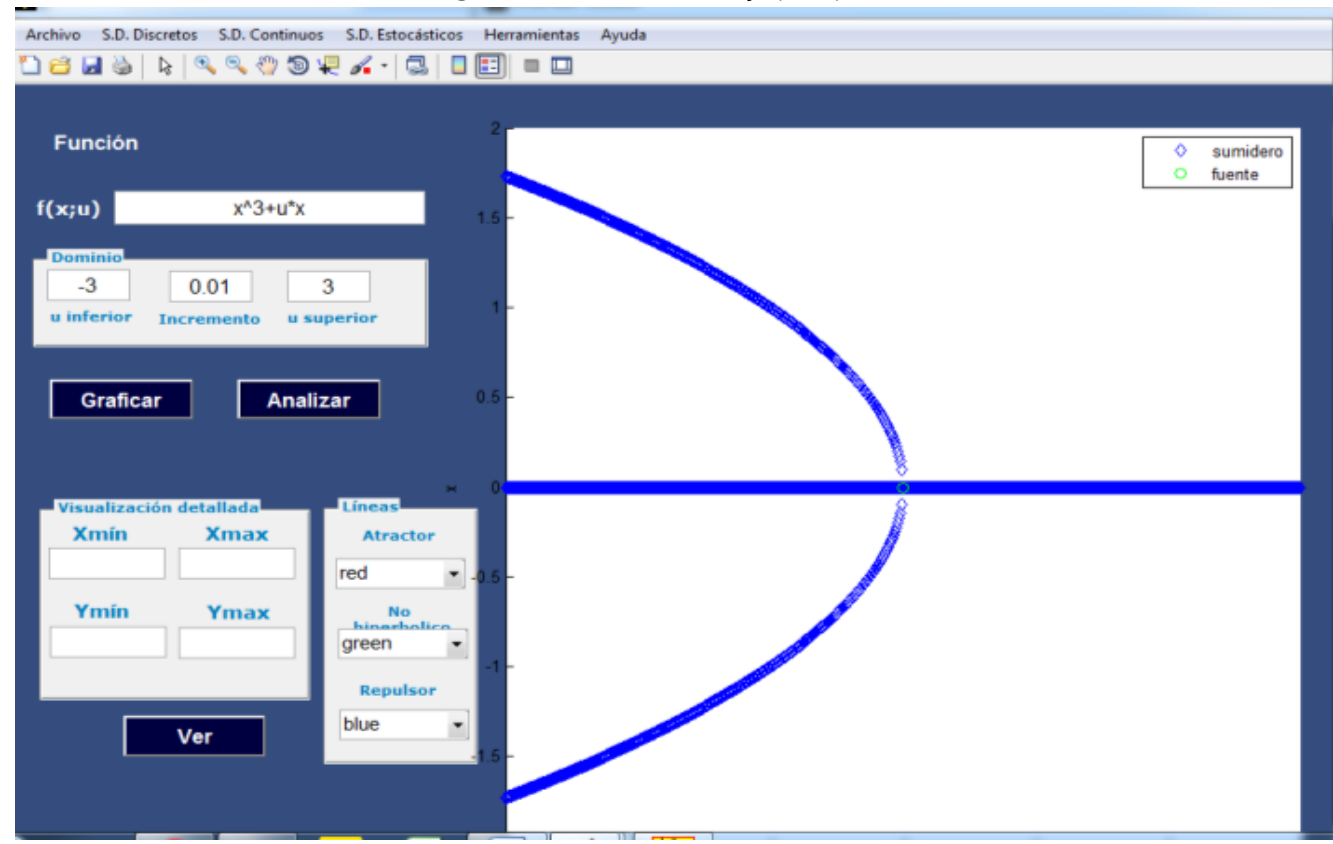

Fuente: Dsamala Toolbox-2 
Figura 21

Descripción del análisis de bifurcación obtenida del sistema $f(x, u)=x^{3}+u x$

Web Browser - file:///D:/Betty/I-215/investigaciones/samala/DSamala/puntosb.txt

puntosb.tat $\times$ +

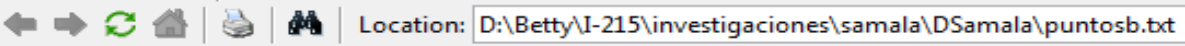

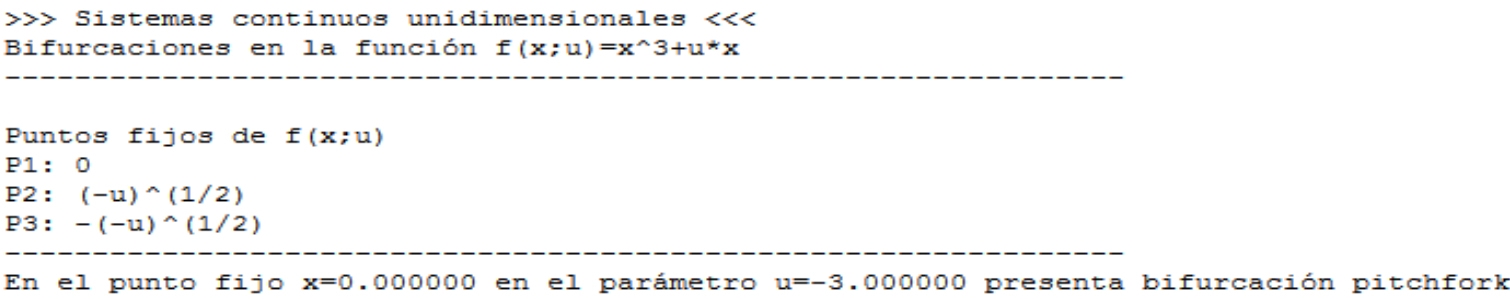

Fuente: Dsamala Toolbox-2

Figura 22

Diagrama de bifurcación pitchfork de la función $f(x, u)=x^{3}+u x$

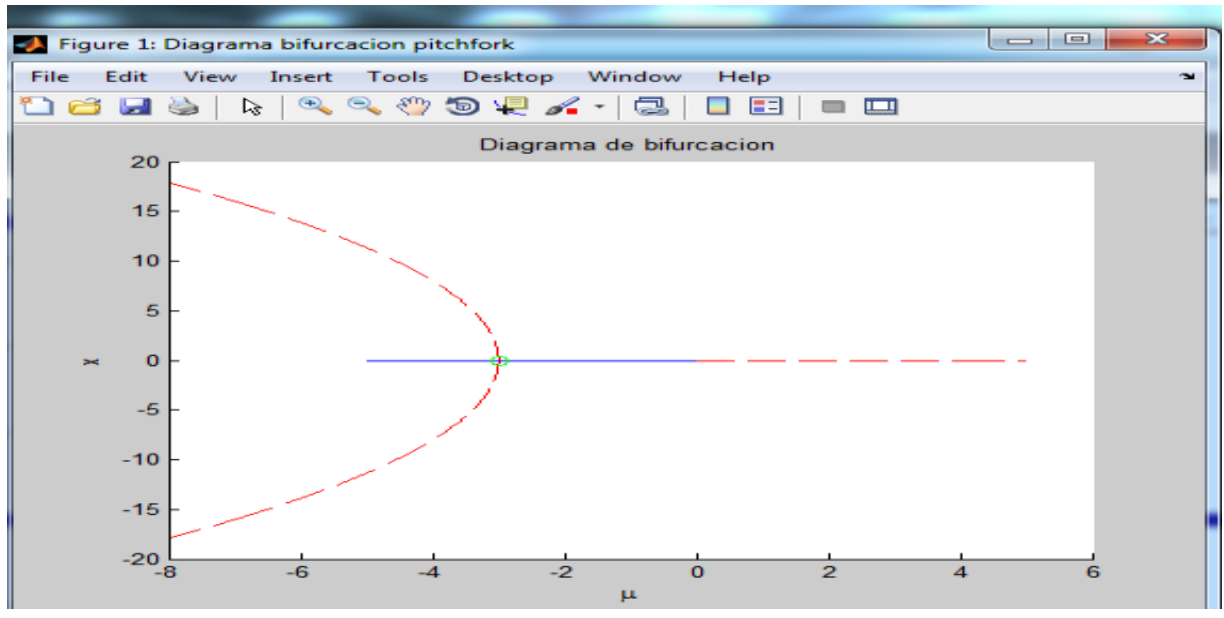

Fuente: Dsamala Toolbox-2

La familia uniparamétrica $f(x, u)=u x-x^{3}$ presenta una bifurcaci $\mathrm{n}$ pitchfork en el punto de equilibrio $\mathrm{x}=0$, bajo el par metro $u=-2$, esto se evidencia en la Figura 23: análisis de bifurcación por medio de Dsamala Toolbox2.

Figura 23

Descripción del análisis de bifurcación pitchfork de la función $f(x, u)=u x-x^{3}$

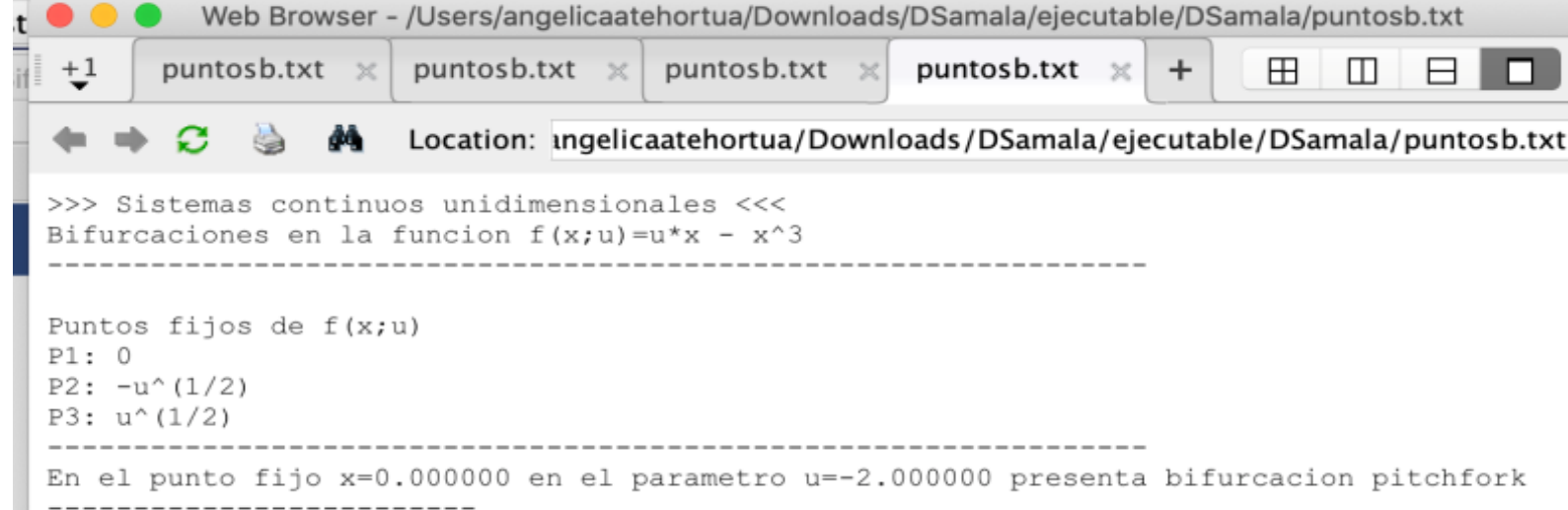
Web Browser - /Users/angelicaatehortua/Downloads/DSamala/ejecutable/DSamala/puntosb.txt

Fuente: Dsamala Toolbox-2 
El sistema $f(x, u)=x-u^{2}-x^{2}$ presenta una bifurcaci $\mathrm{n}$ fold en el punto de equilibrio $x=0,5$; bajo el par metro $u=-0,5$. La Figura 24 muestra el diagrama de bifurcación del sistema y en la Figura 25 se observa el análisis de la bifurcación.

Figura 24

Diagrama de bifurcación pitchfork de la función $f(x, u)=x-u^{2}-x^{2}$

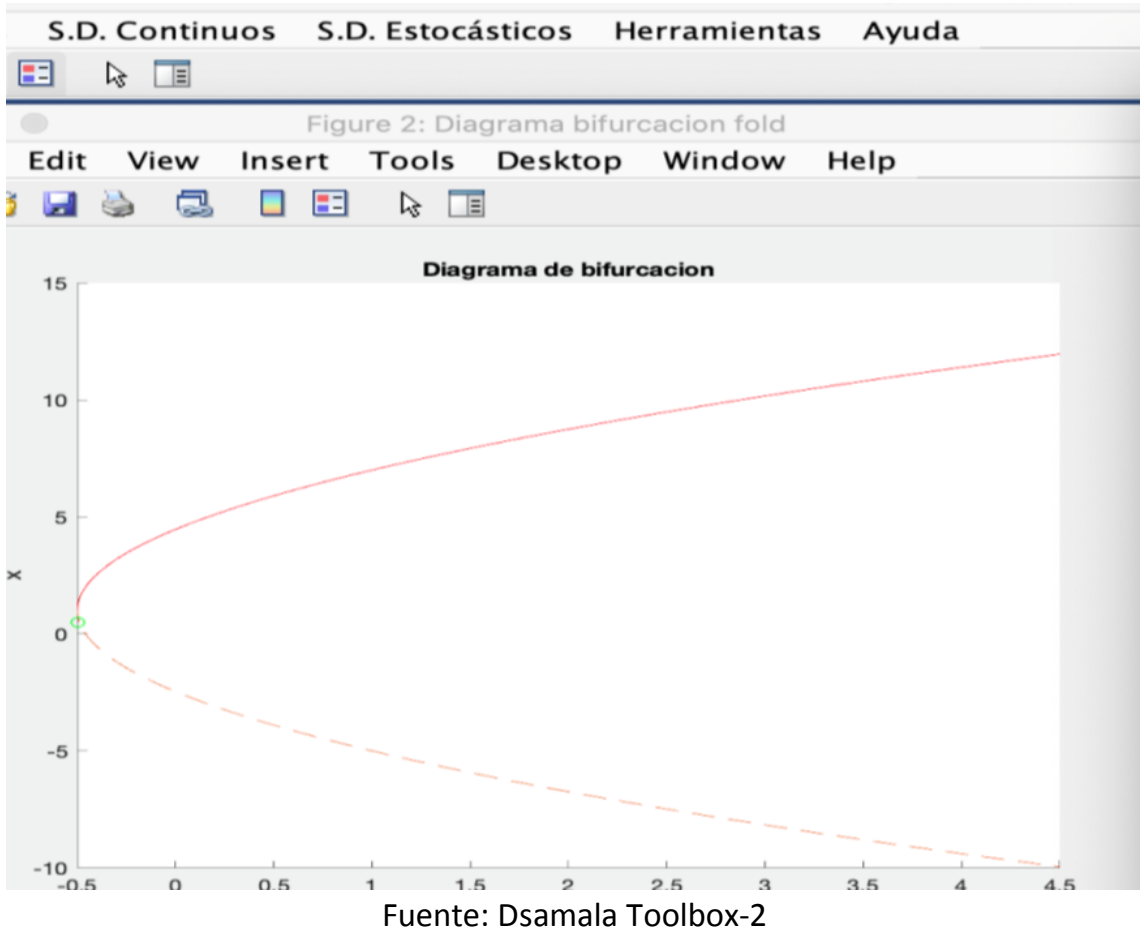

Figura 25

Descripción del análisis de bifurcación pitchfork de la función $f(x, u)=x-u^{2}-x^{2}$ rcaciones Continuos

Web Browser - /Users/angelicaatehortua/Downloads/DSamala/ejecutable/DSamala/puntosb.t

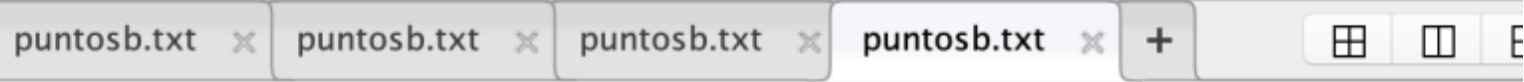

$\Rightarrow \approx$ Location: ingelicaatehortua/Downloads/DSamala/ejecutable/DSamala/pu

>> Sistemas continuos unidimensionales $<<<$

Bifurcaciones en la funcion $f(x ; u)=x-u \wedge 2-x^{\wedge} 2$

Puntos fijos de $f(x ; u)$

P1: $1 / 2-(-(2 * u-1) *(2 * u+1))^{\wedge}(1 / 2) / 2$

P2: $(-(2 * u-1) \star(2 \star u+1))^{\wedge}(1 / 2) / 2+1 / 2$

En el punto fijo $\mathrm{x}=0.500000$ en el parametro u=-0.500000 presenta bifurcacion fold

Fuente: Dsamala Toolbox-2 
Para finalizar, se analiza la familia uniparametrica $f(x, u)=u+x-x^{\wedge} 2$, la cual presenta una bifurcaci $\mathrm{n}$ fold en el punto de equilibrio $x=-0,25$; bajo el par metro $u=-0,5$. En la Figura 26 se visualiza el diagrama de bifurcación y en la Figura 27 se muestra el análisis de la bifurcación mefiante Dsamala Toolbox-2..

Figura 26

Diagrama de bifurcación de la función $f(x, u)=u+x-x^{2}$

Figure 1: Diagrama bifurcacion fold

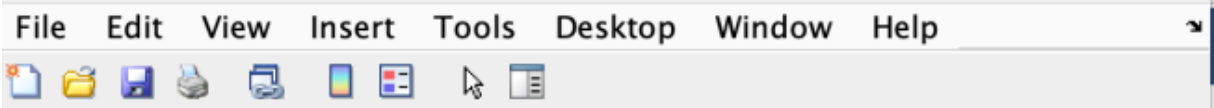

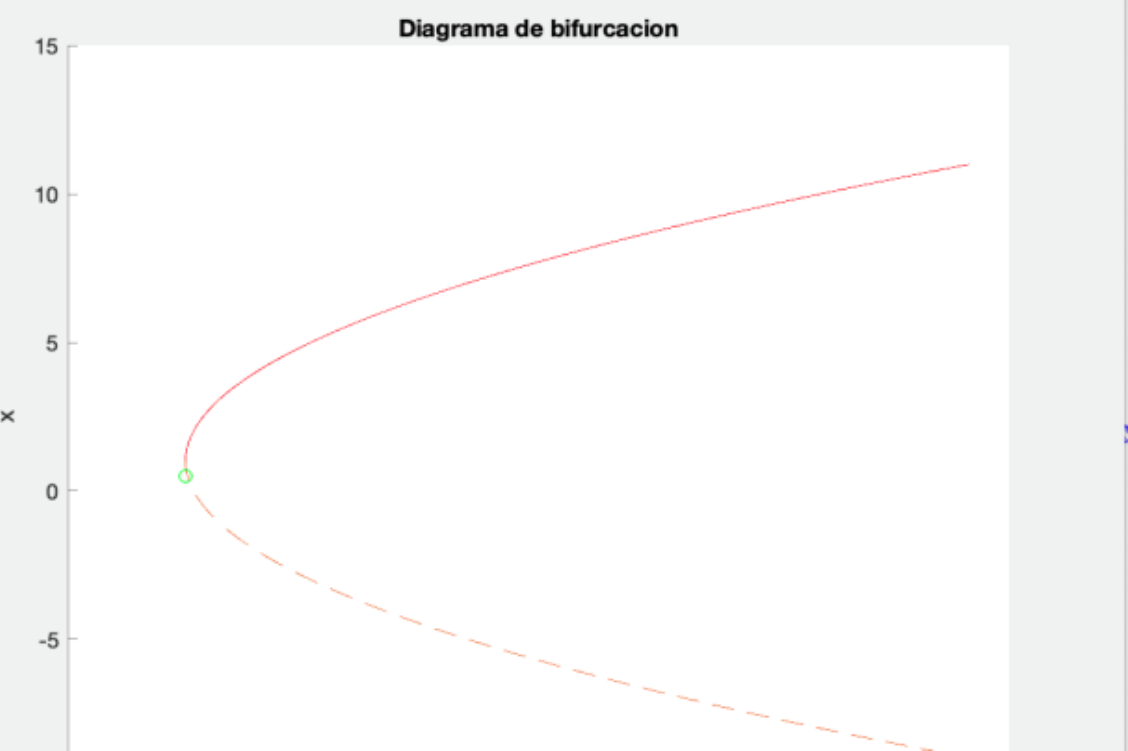

Fuente: Dsamala Toolbox-2

Figura 27

Análisis de bifurcación para la función $f(x, u)=u+x-x^{2}$

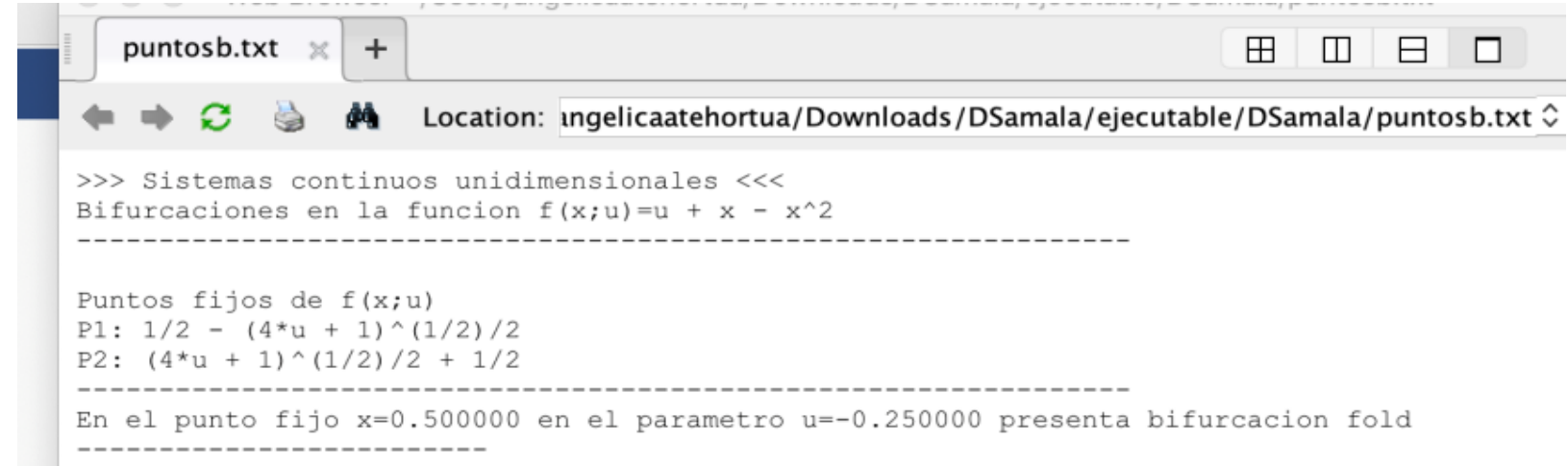

Fuente: Dsamala Toolbox-2 


\section{Conclusiones}

Se ha logrado tener una herramienta que permite determinar bifurcaciones de tipo fold, transcritica, pitchfork y flip en sistemas discretos y bifurcaciones de tipo fold, transcritica, pitchfork en sistemas continuos, bajo condiciones no estándar de bifurcaciones. Para futuras mejoras del software Dsamala Toolbox -2 se puede adicionar que determine otros tipos de bifurcaciones, tanto en sistemas dinámicos autónomos discretos como continuos. Por ejemplo en sistemas continuos se podría agregar que determine bifurcaciones de tipo flip y bifurcaciones Hopf-Neimark-Sacker. También podría extenderse a bifurcaciones en familias multiparamétricas.

\section{Agradecimientos}

Rojas, agradece al Instituto de Investigaciones de la Orinoquia Colombiana, IIOC, de la Universidad de los Llanos por su apoyo para este trabajo a través del proyecto de investigaci $\mathrm{n}$ titulado "Herramienta computacional para el estudio de sistemas dinámicos continuos y discretos". Valverde, agradece a el Ministerio de Economía y competitividad de España bajo el Proyecto MTM2014-51891-P y a FEDER OP2014-2020 de Castilla la ManchaEspaña bajo el proyecto GI20163581.

\section{Referencias bibliográficas}

Atehortúa, A. M., Ladino, L. M., \& Valverde, J. C. (2012). Dsamala toolbox software for analysing and simulating discrete, continuous, stochastic dynamic systems. Ingeniería e Investigación, 32(2), 51-57.

Balibrea, F., Martinez, A., \& Valverde, J. C. (2010). Local bifurcations of continuous dynamical systems under higher order conditions. Applied mathematics letters, 23(3), 230-234.

Balibrea, F., Oliveira, H. M., and Valverde, J. C. (2017). Topological equivalences for one-parameter bifurcations of scalar maps. Journal of Nonlinear Science, 27(2):661-685.

Balibrea, F., \& Valverde, J. C. (2001). Structural stability under conditions of nonhyperbolicity. Computers \& Mathematics with Applications, 41(5-6), 757-768.

Balibrea, F., \& Valverde, J. C. (2003). Cusp and generalized flip bifurcations under higher degree conditions. Nonlinear analysis, 52(2), 405-419.

Dhooge, A., Govaerts, W. y Kuznetsov, YA (2003). MATCONT: un paquete MATLAB para el análisis de bifurcación numérica de EDO. Transacciones ACM en software matemático (TOMS), 29 (2), 141-164.

Domínguez, E., Ardila, F., \& Bustamante, S. (2010). System-Solver: Herramienta de código abierto para la modelación de sistemas dinámicos. Ingeniería e Investigación, 30(3), 157-164.

Elaydi, S., Luís, R., and Oliveira, H. (2013). Local bifurcation in one-dimensional nonautonomous periodic difference equations. International Journal of Bifurcation and Chaos, 23(03):1350049.

Glick, J., Kocak, H., Phaser. [Online]. [Access date: April 2016]. Available at: http://www.phaser.com/

Govorukhin, V. (2011). Obtenido de www.math.rsu.ru/mexmat/kvm/matds/

Guckenheimer, J., \& Holmes, P. (1983). Nonlinear oscillations, dynamical system, and bifurcations of vector fields. Sprinfer.

Kuznetsov, Y. A. (2004). Elements of applied bifurcation theory. New York: Sprinfer.

Polking, J. [Online]. [Access date: April 2016]. Available at: http://math.rice.edu/ dfield/dfpp.html

Tovar, A. (2000). Herramienta para la detección de bifurcaciones locales en sistemas dinámicos no lineales . Bogota: Universidad Nacional de Colombia.

Esta obra está bajo una Licencia Creative Commons

Attribución-NoCommercial 4.0 International 